\title{
Sistema Prefabricado de Ecofachada Termoaislante para el Mejora- miento de Viviendas Sociales Construidas en la Zona Árida Centro Oeste de Argentina
}

\author{
Pre-Made System of a Thermal Insulating Eco-Facade for the Improvement of So- \\ cial Houses Built in Arid Zone West Center of Argentina
}

$\underline{\text { Arturo Florentino Buigues Nollens }}^{(*)}$

\section{RESUMEN}

En este trabajo se desarrolla un Sistema Prefabricado de Ecofachada Termoaislante Sustentable-SPETS, para el mejoramiento del confort interior de Viviendas Sociales Construidas-VSC, en el clima árido de San Juan, Argentina. La metodología considera estudios teóricos y prácticos, sobre las cualidades termofísicas de fachadas opacas del Barrio Aramburu, cumplimiento a Normativas, junto a una propuesta alternativa con componentes estructurales móviles y desarmables incluyendo pomeca puzolánica, como material regional termoaislante ignífugo con origen volcánico. Los resultados obtenidos en el laboratorio IMA-FI-UNSJ, durante las campañas representativas de mediciones invernales, verificaron con el SPETS-sin ventilar una variación entre exterior e interior de $18^{\circ} \mathrm{C}$ $\left(4.12^{\circ} \mathrm{C}\right.$ Capa Externa y $22.15^{\circ} \mathrm{C}$ Revoque Interno), y en verano con casi $47^{\circ} \mathrm{C}$ con el SPETS-ventilado se produjeron diferencias de la capa externa y el revoque externo sin ventilar de hasta $15,5^{\circ} \mathrm{C}$. El SPETS contribuye eficientemente al confort térmico y ambiental con cumplimiento a las Normas Nacionales de Reciclado.

Palabras clave: Sistema Prefabricado; Ecofachada; Construcción Sostenible; Viviendas Sociales Construidas; Zona Árida Centro Oeste Argentina.

\section{ABSTRACT}

In this paper a Pre-made Thermal Insulating Sustainable Eco-facade System - PTISES is developed for the improvement of interior comfort in Built Social Houses - BSH-in the arid climate of San Juan, Argentina. The methodology considers theoretical and practical studies on the thermo-physical qualities of opaque facades in the Aramburu neighbourhood, according to standards, together with an alternative proposal for its improvement built with structural mobile and removable components. This includes pomeca puzolanica as a regional thermal insulating fire retardant material of volcanic origin. The results obtained in the laboratory at the IMA-FI-UNSJ, during representative campaigns of Winter measurements, verified for PTISES-without ventilation a variation of $18^{\circ} \mathrm{C}$ between outdoors and indoors temperature $\left(4.12^{\circ} \mathrm{C}\right.$ External Layer and $22.15^{\circ} \mathrm{C}$ Internal Plaster), and in Summer almost $47^{\circ} \mathrm{C}$ PTISES-with the ventilated. Differences of upto $15,5^{\circ} \mathrm{C}$ between the external layer and the external plaster without ventilation were produced. The PTISES contribute to efficiently the thermal and environmental comfort, under the Standards of National Recycling Plans.

Keywords: Pre-made System; Ecofacade; Sustainable Construction; Built Social Houses; Arid Zone West Center Argentina.

(*) Área de Energías Renovables y Ambiente (AERA) - Instituto de Mecánica Aplicada (IMA) - Facultad de Ingeniería (FI) Universidad Nacional de San Juan, San Juan (UNSJ) - (Argentina).

Persona de contacto/Corresponding author: abuigues@unsj.edu.ar (A. Buigues Nollens)

ORCID: https://orcid.org/oooo-0002-3426-2506 (A. Buigues Nollens)

Cómo citar este artículo/Citation: Buigues Nollens, Arturo Florentino (2021). Sistema Prefabricado de Ecofachada Termoaislante para el Mejoramiento de Viviendas Sociales Construidas en la Zona Árida Centro Oeste de Argentina. Informes de la Construcción, $73(561)$ : e377. https://doi.org/10.3989/ic.74740

Copyright: (C) 2021 CSIC. Este es un artículo de acceso abierto distribuido bajo los términos de la licencia de uso y distribución Creative Commons Reconocimiento 4.0 Internacional (CC BY 4.0). 


\section{INTRODUCCIÓN}

En la Cumbre 2015 para el Desarrollo Sostenible, los Estados Miembros de la ONU aprobaron la Agenda, que incluye un conjunto de Objetivos de Desarrollo Sostenible (ODS), en los que se considera para 2030 la necesidad del mejoramiento de la vivienda, junto con el fin de mejorar la sostenibilidad de los asentamientos humanos rurales y urbanos (1).

Con el objetivo de obtener el reciclado energético de las Viviendas y Departamentos Sociales Construidos (VDSC) (2), se hace necesario e imperioso de implementar una adecuada envolvente, que logre un armonioso diálogo con el clima del lugar y un diseño bioclimático para un correcto comportamiento térmico-energético (3).

En el mejoramiento que se propone realizar a través de este trabajo, se tiene en cuenta la reutilización de materiales de descarte con origen natural, y considera la búsqueda de materiales alternativos para el aislamiento térmico de VDSC, reutilizando recursos de desechos existentes. Otros investigadores, en un anterior trabajo, realizaron una evaluación cuali-cuantitativa de aislaciones térmicas alternativas para viviendas, obteniendo mediciones de los materiales más representativos agrupados en cuatro categorías: fibras naturales, plásticos, papel y en la tierra consideraron a la piedra pómez (4).

También explicitaron que, decisiones tomadas con relación a la eficiencia y el consumo energético del edificio, el tipo de sistema de fachada elegido y sus materiales, así como su correcto diseño y ejecución, condicionarán en gran medida el comportamiento global del conjunto. En este sentido destacaron, que en las fachadas convergen ciertos factores que favorecen la dinámica del incendio: infinita provisión de oxígeno, por su ubicación en contacto con el exterior, la verticalidad de la superficie, su configuración geométrica y el viento entre otros (5).

Por otro lado el devastador incendio sucedido en el 2017, en la Torre Grenfell de Londres con el trágico resultado de más de 80 personas fallecidas, ha motivado que los españoles dirijan la vista al momento de rehabilitar sus viviendas construidas antes de los años 8o, hacia otro requisito de diseño imprescindible, de los distintos sistemas de aislamiento térmico por el exterior de fachadas ventiladas, que cuentan con elementos componentes o materiales altamente inflamables y que han provocado graves incendios ocasionando daños irreparables a la vida de sus usuarios además de generar un alto impacto ambiental (6).

Anteriormente el autor de este trabajo se ha planteado, para el estudio térmico a nivel de laboratorio, la realización de una envolvente con paneles prefabricados termoaislantes, utilizando desechos de piedra pómez llamada pomeca puzolanica (PP) cemento y agua, destinado al acondicionamiento higrotérmico natural de VDSC de zonas urbanas áridas. Los paneles prefabricados termoaislantes de reducidas dimensiones de $0.40 \mathrm{~m} \times 0.40 \mathrm{~m} \times 0.05 \mathrm{~m}$ conformaron una nueva envolvente externa de bajo costo, con el objeto de disminuir pérdidas térmicas (7). Posteriormente en el III Congreso Internacional de Estudios del Desarrollo realizado en el 2017 Zaragoza España, investigadores del Instituto de Ciencia y Tecnología del Hormigón, Universitat Politècnica de Valèn- cia (Icitech-Upv) - España y el Instituto de Mecánica Aplicada, Facultad de Ingeniería, Universidad Nacional de San Juan - Argentina, presentaron un trabajo de investigación conjunta en el que aprovecharon a la PP por sus propiedades puzolánicas, como sustituto parcial del cemento portland en la elaboración de morteros y hormigones, reduciendo así el consumo energético y también el de materias primas (8).

Este trabajo, presenta como objetivo brindar el necesario mejoramiento al confort térmico interior de las VDSC, que les falto incluir en el diseño original de sus fachadas opacas verticales de sus envolventes, considerando las condiciones climáticas externas y el cumplimiento de las Normativas Argentinas. Para obtener el mencionado mejoramiento propone el desarrollo a escala piloto de un Sistema Prefabricado de Ecofachada Termoaislante Sustentable (SPETS), con bajo impacto ambiental que integra la reutilización durante y al final de su ciclo de vida de los materiales y componentes constructivos a utilizar.

Así el desarrollo del SPETS brinda respuestas en relación a su comportamiento global, para ello tiene en cuenta procesos de obra controlados, con mínima producción de residuos. Tanto para la nivelación del muro "soporte" donde se aplica un revoque termoaislante preparado en húmedo, como durante la prefabricación y fijación en seco de módulos livianos multicapas, construidos con componentes estructurales metálicos móviles y desarmables, que incluyen PP a granel como material aislante térmico e ignífugo por su origen volcánico y regional.

\section{METODOLOGÍA EMPLEADA, ESTRATEGIAS, Y MATERIALES}

Considerando la tecnología de construcción utilizadas en las fachadas de las VDSC y los requerimientos que estas presentan para poder resolver la necesidad de acondicionamiento térmico junto a la conservación de la energía interior, la metodología empleada tiene en cuenta los siguientes estudios teórico-prácticos sobre la envolvente opaca:

\subsection{Relevancia del caso en estudio y su entorno.}

\subsubsection{Caracterización Climática de la Región donde se locali- za el estudio:}

El estudio se desarrolla en la Ciudad de San Juan - Argentina, pero es aplicable a VDSC de otros departamentos con gran número de habitantes del Gran San Juan y de la Zona Árida de Cuyo. Como se menciona y recomienda en la Norma IRAM 11603 de Acondicionamiento Térmico de Edificios, el Clima del lugar se caracteriza por tener grandes amplitudes térmicas en el que es aconsejable el uso de estrategias bioclimáticas, tal como las viviendas agrupadas y de todos los elementos y/o recursos que tiendan al mejoramiento de la inercia térmica.

La clasificación bioambiental que le corresponde a la región donde se localiza el estudio, es la Zona III: Templada Cálida. En este sentido la Norma se refiere en forma específica, a que por tratarse de una zona bioclimática templada, las exigencias deben ser menores tanto en la faz de la orientación como en las necesidades de ventilación. Y explicita que para las edificaciones con orientaciones desfavorables, se aconseja prever protecciones solares adecuadas (9). 
En esta región, los veranos son relativamente calurosos y presentan temperaturas medias que oscilan entre $20^{\circ} \mathrm{C}$ y $26^{\circ} \mathrm{C}$, con máximas medias que superan $\operatorname{los} 30^{\circ} \mathrm{C}$, sólo en la faja de extensión Este-Oeste. El invierno no es muy frío y presenta valores medios de temperatura entre $8^{\circ} \mathrm{Cy} 12^{\circ} \mathrm{C}$, y valores mínimos que rara vez son menores que $0^{\circ} \mathrm{C}$. Las presiones parciales de vapor de agua son bajas durante todo el año, con valores máximos en verano que no superan en promedio, los $1870 \mathrm{~Pa}(14 \mathrm{~mm} \mathrm{Hg}$ ).

En general, en esta Zona árido-seca y dentro de la Subzona IIIa, que cuenta con amplitudes térmicas mayores a $14^{\circ} \mathrm{C}$, debido a las situaciones límites que se presentan, en las que se tienen inviernos relativamente benignos con veranos no muy calurosos, es necesaria la racionalización de los recursos, entre ellos el agua (10).

Los vientos predominantes frescos y algo cargados de humedad son los provenientes del cuadrante sureste. El zonda es un viento fuerte, cálido y muy seco asociado a una masa de aire que al descender por las laderas orientales de la Cordillera de los Andes se comprime adiabáticamente. Este evento ocurre con mayor frecuencia en las temporadas de invierno y primavera, principalmente en las provincias de Mendoza y San Juan, en Argentina. El viento Zonda es un viento, local seco y cálido que frecuentemente sopla y lleva mucha suciedad sobre las estribaciones orientales de la Cordillera de los Andes. Este viento nace en el anticiclón del océano Pacífico, por lo tanto inicia siendo un viento frío y húmedo. Luego es desviado por el ciclón de la Precordillera e ingresa en la zona de mayores alturas, y choca con estas generando lluvias orográficas y nevadas en la cima de la cordillera. Luego ingresa en forma seca, y baja rozando la cordillera aumentando su temperatura hasta $40{ }^{\circ} \mathrm{C}$, por el recalentamiento adiabático o efecto Föehn.

En regiones pobladas, se produce una serie de daños en función de la intensidad de las ráfagas del viento con categorías de moderado inferiores a $65 \mathrm{~km} / \mathrm{h}$, severo de entre 65 y 90 $\mathrm{km} / \mathrm{h}$, muy severo y con ráfagas que van desde los 90 hasta los $120 \mathrm{~km} / \mathrm{h}$ y extremadamente severo o catastrófico donde las ráfagas ya superan $\operatorname{los} 120 \mathrm{~km} / \mathrm{h}$ (11).

El fuerte viento, sequedad, altas temperaturas y la fricción entre partículas de polvo favorecen en forma directa a la generación y propagación de incendios en viviendas, causa daños a la interface o áreas con pastos secos y de cultivos. Y además de generar la evacuación de personas y animales, provoca voladura de techos, caída de árboles y ramas. En forma indirecta origina daños en instalaciones de gas y líneas eléctricas de alta tensión, con interrupción de los sistemas de comunicación (12).

La Dirección Provincial de Defensa Civil de Mendoza hizo público en un Diario local, el parte numérico con 1045 novedades que se produjeron con el paso del Viento Zonda durante el pasado 21 de julio de 2019 y que también influyera con similares daños a San Juan, Tabla 1 (13). Cabe destacar que el impacto ambiental que se originara en el entorno incluyo incendios que afectaron a varias viviendas en esta zona de Cuyo. Por ello resulta de interés para este trabajo, utilizar materiales en las fachadas que no favorezcan la propagación del fuego, debido a que la interface de esta zona árida es vulnerable a la ignición (14).

\subsubsection{Descripción tipológica y tecnológica de las envolventes verticales de VDSC:}

El caso adoptado como característico, que se analiza en este trabajo como representativo de las VDSC es el del Barrio Aramburu. Comenzó a habitarse en 1983 y tiene 1.256 viviendas entre casas y departamentos. Según la división Agrimensura del IPV, el Aramburu cuenta con una extensión de 45 ha, viven más de 7 mil personas, y es uno de los barrios sanjuaninos con mayor cantidad de unidades habitacionales construidas. Cabe destacar que sus fachadas opacas verticales, fueron construidas con bloques de hormigón sin revoques por el exterior.

Tabla 1. Novedades producidas en la zona árida de Mendoza Argentina con el Viento Zonda, durante el 21 de julio de 2019 (13).

\begin{tabular}{|c|c|c|c|c|c|c|c|c|c|c|}
\hline Novedades & & & Parte & Numéric & - Dirección & Provincial de D & fensa Civil & Mendoza & & \\
\hline & & Ince & ndios & & Fyocuados & Derrumbe & Voladura & Árbol/ & Varios & Total \\
\hline Departamento & Vivienda & Forestal & Interface & De Cerro & Evacuados & de Viviendas & de Techo & Rama Caído & $(*)$ & \\
\hline Capital & & & & & & & 05 & 41 & 13 & 59 \\
\hline Godoy Cruz & & & & & & & 03 & 22 & 15 & 40 \\
\hline Guaymallén & & & & & & 08 & 13 & 207 & 77 & 305 \\
\hline Las Heras & & 01 & 01 & 01 & & & 04 & 173 & 85 & 265 \\
\hline Luján & 34 & 01 & & & 60 & & 70 & 62 & 06 & 233 \\
\hline Lavalle & & & 08 & & & & & & & 08 \\
\hline Maipú & & & & & & 02 & 03 & 14 & 11 & 30 \\
\hline Rivadavia & & & 02 & & & & & 02 & & 04 \\
\hline San Carlos & & & 06 & & 04 & & & 27 & 08 & 45 \\
\hline San Martín & & & 01 & & & & & 01 & & 02 \\
\hline Tupungato & & & & & 50 & 04 & & & & 54 \\
\hline Total & 34 & 02 & 18 & 01 & 114 & 14 & 98 & 549 & 215 & 1045 \\
\hline
\end{tabular}

Observaciones: ${ }^{*}$ ) incluye Fugas de Gas, Transformadores en Corto, Cables Cortados o en Corto, Postes Caídos y Apoyo Hídrico. Cabe aclarar que los Departamentos de la zona árida de Mendoza que No reportaron novedades no fueron incluidos en esta tabla. 
La superficie de este conjunto no fue proyectado para ser autoconstruido, pero dada las características porosas que ofrecen las fachadas, los usuarios de los departamentos utilizan cualquier oportunidad de aprovechamiento de espacios semiexteriores, para realizar crecimientos progresivos no proyectados, dentro de su estructura y/o en algunos casos fuera de ella (15).

En este Barrio, claramente se observa como la evolución y adaptación en el tiempo de la vivienda es un proceso indisoluble de la vida cotidiana y el "ciclo de vida vital". Esta evolución incluye a la necesidad de identificación que sienten sus habitantes, de personalizar el ambiente en el que viven (16).

Es decir que la estructura de la vivienda o departamento existente es un "soporte", qué igualmente evoluciona o crece según las necesidades espaciales familiares, y se muestra como que nunca está completamente terminada, a pesar de no ser un conjunto con genética adaptable.

Esta situación puede observarse en la Figura 1a, donde se muestra como los usuarios de los bloques de departamentos en altura han avanzado sobre planta baja y balcones, incluyendo ampliaciones con ladrillón visto.

$\mathrm{Al}$ realizar estas modificaciones sin planificación, sobre espacios exteriores de accesos o zonas de servicios y zonas de expansión, no solo han eliminado los espacios de transición exterior - interior proyectados, sino que han anulado la posibilidad de aprovechar la necesaria ventilación cruzada natural que poseían los ambientes interiores. Y eliminado tanto el control de la radiación solar a través de los aleros, como la exposición de los usuarios a la ventilación natural, durante el periodo estival.

También se visualiza en las Figuras $1 \mathrm{a}, 1 \mathrm{~b}$ y 1c, como han pintado con diferentes colores el conjunto, e instalado en forma arbitraria diferentes equipos de aire acondicionado, afectando su imagen estética.
Por otro lado en Figura 1d y 1e, se observa como los usuarios de viviendas en planta baja, revisten las fachadas opacas verticales solo por razones estéticas, sin considerar la carga térmica que influye en sus interiores, debido a la alta absorción solar, respecto del color de la superficie y características del acabado del revestimiento cerámico, aplicado sobre la fachada original.

\subsection{Análisis del comportamiento térmico de facha- das existentes y propuesta de mejoramiento.}

A los efectos de concretar los objetivos propuestos, se analizan los resultados de aplicar la línea 11600 de la Normativa IRAM con el Software de Diseño Higrotérmico (SDH) (17), a la Fachada en la Situación Original y a la Propuesta como Situación Mejorada.

Tal como se describiera, la situación original del $\mathrm{B}^{\circ}$ Aramburu, consiste en muros de bloques de hormigón visto, que determinan una fachada modular con estructuras de hormigón visto y revoque interior.

En estas VDSC, se ha intentado resolver el confort térmico teniendo en cuenta solo en sus cubiertas de techo, un complejo aislante de cemento, arena y poliestireno expandido en copos. Sin considerar materiales y colores del resto de la envolvente opaca vertical. Donde en casi todos los casos, los muros de las fachadas de sus envolventes, son de bloques de hormigón y estructura de hormigón armado, vistos. Si se asume que su conductividad térmica es obtenida mediante una pared de un espesor de $0,18 \mathrm{~m}$ y revoque interior de yeso a la cal $0,025 \mathrm{~m}$, se alcanza una Transmitancia Térmica U de $2,39 \mathrm{~W} / \mathrm{m}^{2} \mathrm{~K}$.

Tanto en el proyecto del Barrio Aramburu, como en otros conjuntos con estas características constructivas realizadas por aquellas décadas como en la actualidad, por el Instituto Provincial de la Vivienda (IPV), no se cumple con la transmi-

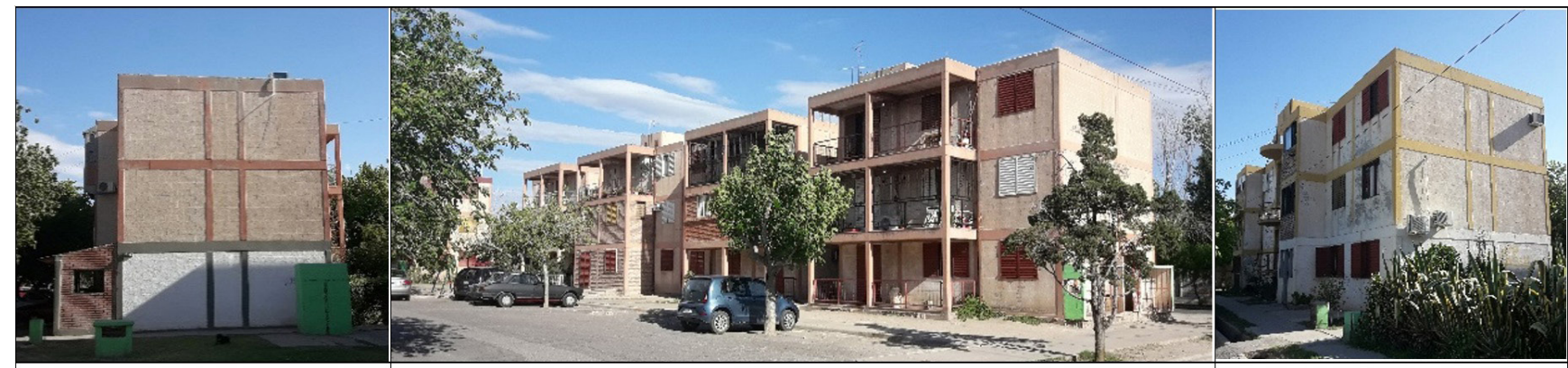

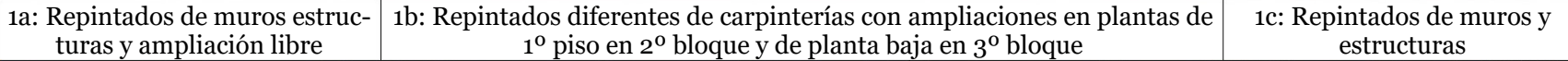

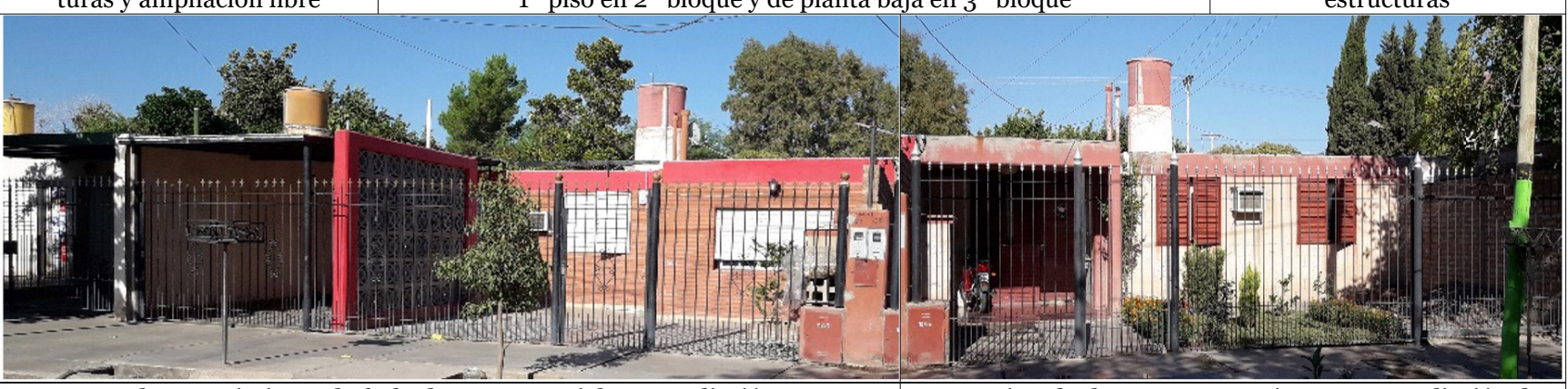

1d: Revestimiento de fachadas con materiales y ampliación de cochera con colores de alta absortividad solar 1e: Repintado de estructuras existentes y ampliación de cochera con color de alta absortividad solar

Figura 1: Caso Barrio Aramburu. Modificaciones realizadas por los usuarios. Fuente: Elaboración del autor. 
tancia térmica máxima para verano o invierno (Umax), en el Nivel C, ya que se requiere un Umax de $1,71 \mathrm{~W} / \mathrm{m}^{20} \mathrm{~K}$.

Y mucho menos para una vivienda social pensada con conservación energética del Nivel $\mathrm{B}$, que requiere un Umax de 0,96 $\mathrm{W} / \mathrm{m}^{2} \mathrm{~K}$ admitida por la Normas del Instituto Argentino de Racionalización de Materiales - IRAM (18).

Por lo tanto esta situación en la que No se Verifica la utilización de estos muros para verano ni invierno, es común en casi todas las fachadas verticales opacas de las VSDC, que con similares diseños fueron construidas posteriormente al del Aramburu, en diferentes zonas de San Juan.

Por lo tanto, producto de una situación constructiva con baja calidad térmica que es generalizada en estas fachadas, sus usuarios soportan críticas situaciones de habitabilidad, vinculadas a la rigurosidad climática estacional.

A lo que se suma, que estas envolventes presentan orientaciones que coinciden con las de máximo asoleamiento, donde la alta penetración solar produce un alto impacto con gran contribución calórica y que junto al escaso o nulo movimiento de aire, hacen que se agrave la situación de disconfort interior producto de la acumulación del calor.

En este sentido la IRAM 11601, permite no tener en cuenta el efecto acumulativo o de inercia térmica, y solo se encuentra principalmente dirigida a estudiar el problema de transmisión de calor de la envolvente, bajo el punto de vista de la resistencia térmica, con flujos de calor predominantemente por conducción.

En la que, para poder conocer el comportamiento térmico de sus elementos o sistemas constructivos, se aplica una metodología de balance térmico, que incluye las características térmicas de los materiales, con un régimen estable o estacionario (18).

Por otro lado de la IRAM 11603, se puede extraer claramente para las regiones cálidas, cuales son las orientaciones térmicamente desfavorables en verano. Por esto, el análisis de la propuesta de mejoramiento que considera al SPETS con sus componentes y que se desarrolla en este trabajo, se encuentra dirigida a brindar respuesta a las orientaciones más desfavorable tal como es la Este u Oeste o con mayor carga térmica e incluye el grado en el que las variantes de la propuesta de diseño para esta fachadas, cumplen con las Normas IRAM mencionadas.

\subsection{Desarrollo de alternativas de diseño consider- ando el confort higrotérmico interior.}

\subsubsection{Análisis de costos energéticos de producción y conducti- vidad térmica de los aislantes utilizados:}

Existe una diversidad de materiales locales, ya sea naturales o de reciclados, que son aptos para mejorar la envolvente de los edificios, con la consecuente mejora del confort y la reducción de la necesidad de consumo de combustibles.

La PP si bien tiene características parecidas a la piedra pómez, también llamada pumita, jal, liparita, posee una composición mineralógica diferente, ya que es una roca ígnea volcánica vítrea de baja densidad $\left(300 \mathrm{~kg} / \mathrm{m}^{3}\right)$, muy porosa de color ocre claro o gris que contiene un alto porcentaje de cuarzo, feldespato, calcita y mica (biotita).

Entre otros muchos usos, dentro de la construcción se emplea tal como ha sido mencionado anteriormente para mejorar las condiciones térmicas y acústicas, y también triturada destinada a la fabricación de morteros u hormigones de áridos ligeros.

El coste energético de producción en los procesos de extracción de materia prima, fabricación, transformación y transporte, que presenta un material aislante de origen volcánico como la Perlita o la PP, son asumidos como bajos (7). Este coste, varía de 5 a $20 \mathrm{MJ} / \mathrm{Kg}$ y posee una conductividad térmica de 0,04 a $0,06 \mathrm{~W} / \mathrm{m}^{\circ} \mathrm{K}$. Mientras que el Poliestireno Expandido según su densidad posee un de 0,029 a 0,053 W/ $\mathrm{m}^{\circ} \mathrm{K}$, y posee altos consumos energéticos durante la fabricación que varían de 75 a $150 \mathrm{MJ} / \mathrm{Kg}$ (19).

\subsubsection{Estudio sobre la disposición del aislamiento térmico usado:}

El elemento arquitectónico del cual dependen en mayor grado el confort y la eficiencia energética de las VDSC, son sus fachadas. Estas aparte de ser la cara estética de la construcción, las Normas mencionadas, permiten emplearlas como una herramienta funcional que contribuye a la sostenibilidad $\mathrm{y}$ funcionalidad de estas edificaciones.

A través de diversos elementos arquitectónicos es posible aprovechar o controlar la radiación solar, con un perfecto equilibrio en la climatización interior, tanto en invierno como en verano (20). Debido a que por lo general en San Juan y la Zona Árida de Cuyo, las VDSC no presentan aislamientos en la envolvente vertical externa o de fachada, por ello el calor tiende a "escaparse" a las zonas más frías para así igualar su temperatura. Esto provoca desplazamientos del aire caliente del interior al exterior en invierno y a la inversa en verano.

Esta deficiencia desde el punto de vista energético, necesita de un importante consumo destinado a la refrigeración o calefacción según la época del año, debido a que en la masa interna no se acumula o conserva la temperatura, ni se detiene su aumento.

Hay una clara diferencia entre la rehabilitación por el interior o por el exterior, y esta diferencia despende de la corrección de los puentes térmicos. De una forma general, por el interior se deben estudiar más a fondo ya que por el exterior, el sistema constructivo es continuo y resolvemos de una manera más sencilla estos puntos conflictivos.

Los puentes térmicos, tiene un papel importante en el balance energético de un edificio, en general, las pérdidas o ganancias térmicas por la fachada supone más del 30\% en edificios poco aislados, y los puentes térmicos lineales pueden suponer más del $30 \%$ de la propia fachada (21).

Esto determina el comportamiento energético de las VDSC (22), debido a que se produce una interacción térmica permanente a través de la envolvente con el exterior, intercambiando calor con el entorno (23). Esta interacción térmica es la que es necesario limitar, disponiendo el aislamiento térmico por la cara externa (24). 


\subsubsection{Estudio de fachadas multicapas a nivel piloto:}

Entre las alternativas prefabricadas estudiadas a nivel teórico del SPETS a la que se dirige esta propuesta, se encuentran las destinadas a las fachadas con alta carga térmica como Sistema termoaislante con Cámara de Aire transventilada exterior, identificado como Sector con Módulo Ventilado, Figuras 2a y 2b. Presenta una Estructura de Soporte que se vincula al muro base revocado con perlita. Posee una Capa Interna que contiene 50mm de PP como material de aislación con origen mineral regional, de origen volcánico que fue dispuesto a granel y en seco.

Y una Capa Externa que es expuesta a la carga térmica de la radiación solar. Esta Capa presenta una cámara de aire de $150 \mathrm{~mm}$ con aberturas a nivel inferior y superior, cuyas hojas se abren o cierran facilitando la ventilación en verano e impidiendo el paso del aire en invierno, Figuras $2 \mathrm{c} \mathrm{y}$ 2d. Tanto la Capa Externa como la Interna y las aberturas móviles mencionadas, son de chapa metálica DD N ${ }^{\circ} 18$.

El conjunto experimental alcanza un espesor total de 240mm, Figura 2b. Esta alternativa fue estudiada con la PP, que si bien Verifica para verano, No Verifica su utilización para invierno, debido a que la transmitancia térmica U del muro, alcanza $1, \mathrm{ooW} / \mathrm{m}^{2 \circ} \mathrm{K}$, superior al $\mathrm{U} \max$. adm. de $0,87 \mathrm{~W} / \mathrm{m}^{2}{ }^{\circ} \mathrm{K}$.

Por ello se optó por la utilización de un tamizado de la PP, realizado con pasos de zarandas de 0,5 y $1 \mathrm{~cm}$ (PPZ), resultando así la posibilidad de alcanzar el valor U deseado para el muro, Figura 3g. Debido a que presenta una mayor granulometría con gran cantidad de cavernas o huecos y microcélulas cerradas o vacías, Figura $3 \mathrm{~h}$. Este material contiene un alto porcentaje de mica (biotita), con una resistencia térmica similar a la Perlita Expandida suelta. Para una densidad entre $30-130 \mathrm{~kg} / \mathrm{m}^{3}$, el valor de la PPZ, alcanza una conductividad térmica $\lambda$ de $0.054 / \mathrm{m}^{\circ} \mathrm{K}$ (25).

\subsection{Construcción e instalación del módulo de prue- ba al aire libre.}

\subsubsection{El SPETS y su adaptación a fachadas existentes:}

Para facilitar la adaptabilidad por deficiencias constructivas entre el SPETS y la construcción existente, o diferencias de verticalidad entre estructuras de hormigón armado y muros de bloques de hormigón, fue necesario realizar un mejoramiento de las fachadas del Laboratorio para ensayos de VSC.

Para ello se colocó como revestimiento un revoque húmedo exterior, en un espesor de $35 \mathrm{~mm}$. Compuesto por un complejo que incluye a la perlita como aislante térmico de origen volcánico. Este, es otro conjunto para ensayos comparativos experimentales identificado como Sector Sin Módulo y sin ventilar, que fue expuesto en forma simultánea al ambiente exterior, Figura $2 b$.

Los materiales aislantes seleccionados son de una materia prima que va de muy poco elaborada a elaborada, y se los ha adoptado por ser derivados de la naturaleza. En el caso de la PP que proviene de las erupciones volcánicas, compuesto principalmente de aluminosilicatos, como resultado de la formación de materiales con estructura desordenada o vítrea con minerales pobremente cristalinos, es enfriado bruscamente en forma natural.

También la perlita es un material aislante con origen volcánico pero con mayor elaboración debido a que es sometido a un proceso industrial mayor antes de ser utilizado en la construcción. Ambos materiales termoaislantes se encuentran exentos de elementos nocivos, facilitan los intercambios de humedad entre la vivienda y la atmósfera. En el caso de la PPZ a granel incluida en el SPETS, es muy poco elaborada y se obtuvo cerca de la obra.

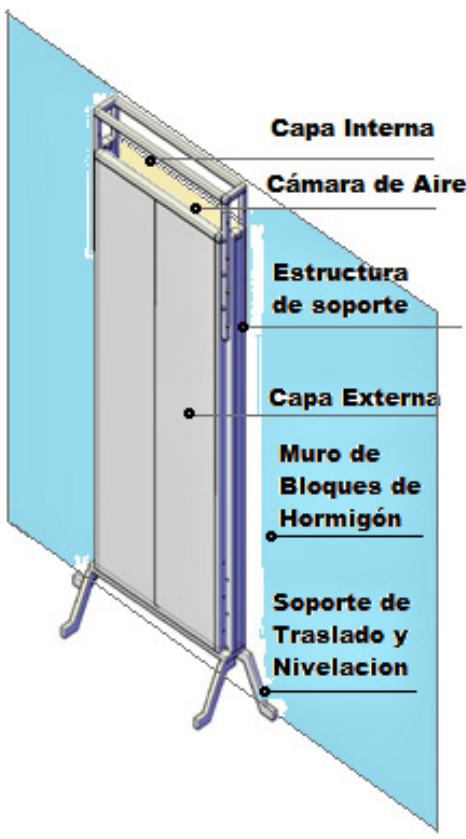

2a: Vista esquemática del SPETS
CONFIGURACIÓN FACHADA

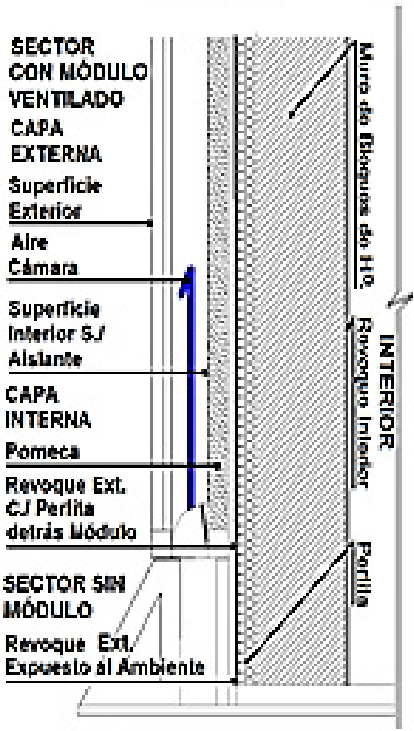

2b: Componentes y funcionamiento del SPETS

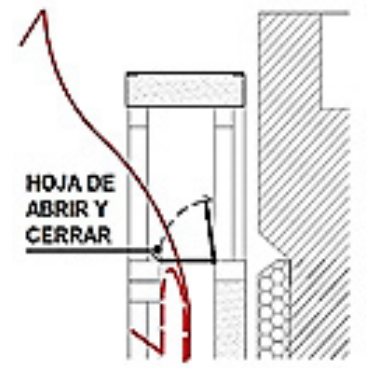

2c: Abertura Superior

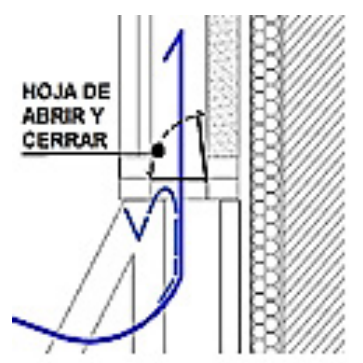

2d: Abertura Inferior 
En estas alternativas se estudiaron además del aislante térmico, la aplicación de superficies protectoras del aislante y la seguridad sísmorresistente (26).

2.4.2. Fabricación de módulos considerando el empleo de procesos de obra controlados con mínima producción de residuos:

Tanto el diseño como la fabricación de las partes del SPETS, consideran la mínima producción de residuos. Por ello el punto de partida del diseño, preserva las dimensiones originales de cada uno de los componentes, Figura $3 a$.

La fabricación incluye la adopción de un racional sistema constructivo en seco, que contempla durante las etapas de prepin- tado, agujereado, ensamble y fijaciones para vinculación de las chapas continuas de las Capas, Externa (Superior) e Interna (Superior e Inferior) al soporte estructural, Figuras 3b, 3c y $3 \mathrm{~d}$.

Tal como se mencionara en el apartado 2.3.3, los módulos de prueba al aire libre fueron construidos con componentes estructurales metálicos livianos, con la concurrencia de materiales convencionales. Y también con la participación de materiales no convencionales derivados de la naturaleza, como la PP mencionada.

Con este aislante que fuera estudiado teóricamente según las necesidades térmicas acordes a la zona árida, fue probado a través de ensayos en Laboratorio con el objetivo de analizar durante el rellenado de Capas, su aplicabilidad junto a la hi-

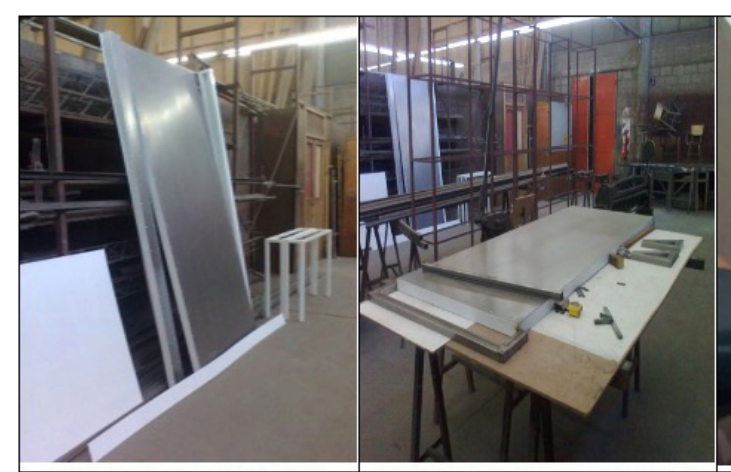

3a: Componentes con pre- 3 b: Prepintado, agujereado 3 servación de dimensiones originales.

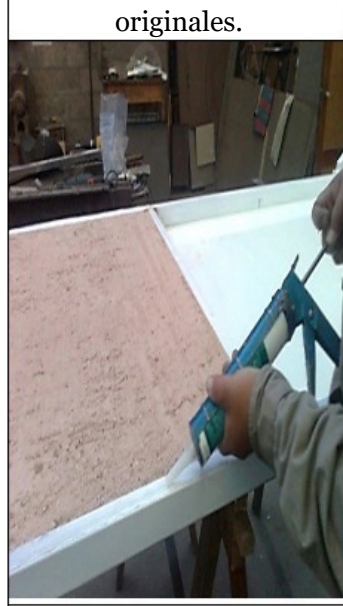
$\mathrm{y}$ armado con fijaciones en seco.
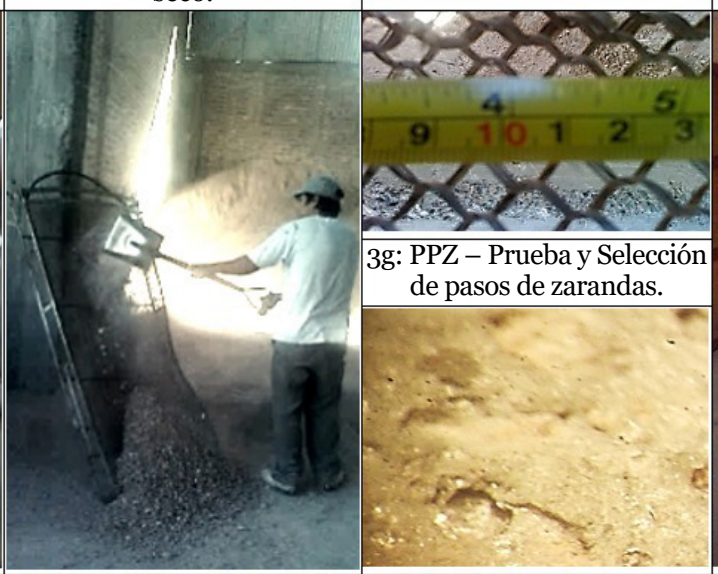

\begin{tabular}{c|c} 
3e: PP-Ensayo Inicial de & 3f: PPZ-Ensayo Posterior de \\
Distribución, compactación & Tamizado y separación de
\end{tabular} y junta selladora en etapas. tierra puzolanica de la PP.

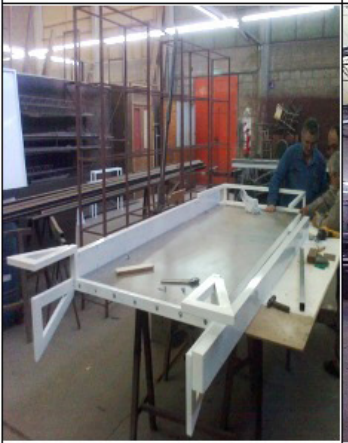

3j: Ensamblado final y fijación de componentes a la estructura de soporte.

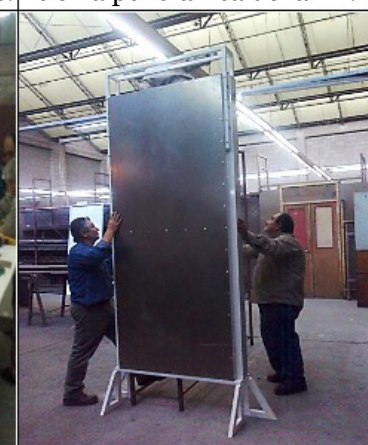

3k: Armado integral del sistema y Limpieza de Capas para prepintado. 3c: Agujereado y fijación de componentes.
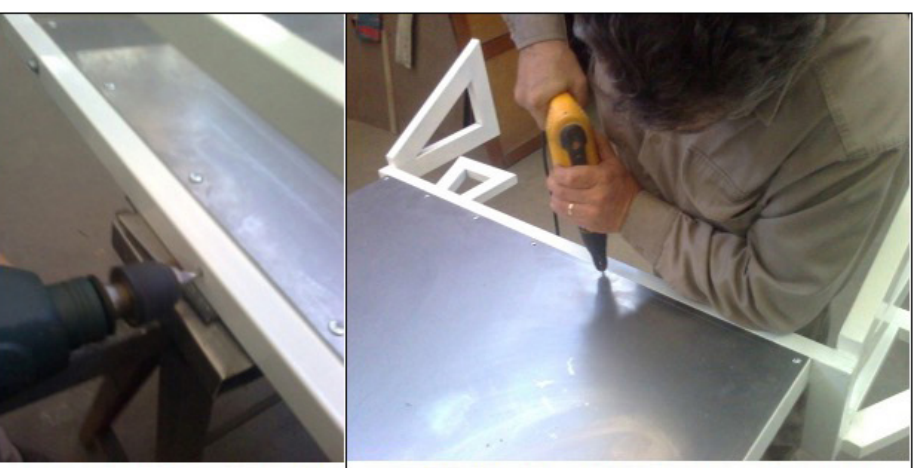

3d: Fijación de las Capas Externa e Interna al soporte estructural para traslado desde la industria metalúrgica y nivelación en la obra.

Figura 3: Desarrollo del estudio de Prefabricación e Instalación en obra. Fuente: Elaboración del autor. 
giene, seguridad y producción de residuos. Durante esta etapa industrial y a modo de Ensayo Inicial, se aplicó en seco la PP suelta a granel, tal como viene de la cantera, Figura 3e .

Requirió la utilización de reglas para la distribución, compactación y barbijos para la protección de los operarios contra el polvo. Y para controlar la voladura de los minerales que contiene, se colocó entre la chapa y el marco estructural una junta selladora estanca, Figura $3 \mathrm{e}$.

Esta situación llevo en un Ensayo Posterior, a someter a la $\mathrm{PP}$ a un proceso de tamizado para la reducción de la presencia de tierra volcánica compuesta por el feldespato y la calcita. Así se disminuyó su densidad junto a la capacidad puzolánica, para aumentar la capacidad como aislante térmico (PPZ), Figura $3 f$.

La prueba con la colocación o rellenado de la Capa Interna con la PPZ, luego de ser zarandeada con el tamizado de $1 \mathrm{~cm}$, Figura 3 f y g, demostró que mejora notablemente su aplicabilidad, debido a que evita la necesidad de protección contra el polvo y juntas en el sistema envolvente, disminuye el peso en casi $50 \%$, facilita la distribución, compactación y relleno en una sola vez, Figura $3 i$.

Y el Ensamblado de Componentes no requiere de sellado de bordes. Lo cual contribuye por un lado a alivianar su traslado y disminuir la sobrecarga de la estructura antisísmica principal de las VDSC, Figura 3 i.

Luego del armado integral del sistema, se realizaron las etapas de limpieza, prepintado y pintado final, considerando en todo el proceso la menor producción de sobrantes y desperdicios encaminado a la reutilización de cada uno de los materiales al final de su ciclo de vida, Figuras $3 \mathrm{j}, 3 \mathrm{k}$ y $3 \mathrm{l}$.

Posteriormente se continuó con la fijación del SPETS, a la fachada nivelada con el revoque aislante. El sistema de fijación que fuera ensayado, atraviesa el mencionado revoque y finaliza anclado sobre hormigón endurecido, Figura 3m. Fue realizado para resistir las enormes fuerzas generadas por vientos muy severos.

La nueva envolvente tiene que resistir las fuerzas laterales directa de estos viento y la succión que este es capaz de provocar sobre el sistema transventilado, junto a las fuerzas de elevación que a veces logran vencer la gravedad.

Este sistema se encuentra compuesto por anclajes de expansión $\mathrm{N}^{\circ} 10$, que se insertan en orificios pre-perforados. Luego se expanden ajustando una tuerca de torque controlado que impide la deformación de la superficie estructural del SPETS, además de facilitar el posterior desarme y reutilización de sus partes.

Así estos anclajes, transfieren la carga de tracción del bulón al hormigón por presiones o fuerzas de expansión mediante fricción o acción de palanca contra la pared de la perforación.

\section{RESULTADOS EXPERIMENTALES}

La construcción original del Laboratorio, presenta características muy similares a una vivienda social mínima del $\mathrm{B}^{\circ}$ Aramburu, con revoque interior, y bloques de hormigón jun- to a estructura de hormigón armado vistos en su envolvente externa. Presenta orientaciones desfavorables de sus fachadas más largas. Los estudios pilotos a nivel práctico, que incluyera la realización de un examen previo del estado de puntos singulares de estas fachadas existentes con la situación térmica más desfavorable, permitió determinar como resultado la necesidad de ejecutar un tratamiento de nivelación mediante un revoque exterior o capa aislante térmico con perlita. Este conjunto constructivo constituye la fachada base y panel con Revoque Exterior sin ventilar.

Cabe destacar que si bien la necesaria adaptación fue realizada con perlita, puede ser totalmente reemplazado por la tierra térmopuzolánica como material de origen volcánico. Esta es obtenida en el proceso de tamizado, en el que es posible la selección de la pomeca aislante térmica pura o PPZ. Ya que este material resultante del zarandeado con una granulometría inferior a los $8 \mathrm{~mm}$, es de un costo muy bajo.

En este proceso se logra aprovechar la doble propiedad intrínseca de la PP, determinada por un lado con las características cementante de los componentes que facilitan la resolución tanto de las faltas de nivelación, como de verticalidad superficial de los muros existentes, para luego poder ejecutar las fijaciones soportes del SPETS; y por otro lado por la característica de aislante térmico e ignifugo.

Ensayos piloto estacionales con el SPETS, realizados durante los años 2016 a 2018 en el Laboratorio IMA-AERA-FI-UNSJ, demostraron adecuadas respuestas de su comportamiento global.

Para facilitar estudios comparativos, se utiliza un sistema de medición integrado en tiempo real, que se caracteriza como instrumento de medición por su simultaneidad, escalabilidad, flexibilidad a distintos parámetros térmicos tales como Temperatura de Aire Exterior y Radiación Solar, Figura 4.

Y el sensado del comportamiento térmico dinámico del sistema y sus variantes en estudio consideraron la obtención de información de las características dinámicas de los materiales componentes de la fachada termoaislante, a través de la búsqueda de mediciones in-situ arriba y abajo del Sector con Revoque Exterior sin ventilar y del Sector de Revoque bajo el Modulo ventilado, Temperaturas de Entrada y Salida del Conducto, Figura 4.

En la Figura 5 se observan resultados obtenidos en la ciudad de San Juan sobre la fachada del laboratorio del IMAFI-UNSJ. De la campaña de mediciones representativa del periodo estival realizada entre el 19-20/2/17, mediante datos obtenidos por la Estación Meteorológica digital WH1081 y un Sistema Adquisitor de Datos Fluke con Sensores LM73 alámbricos, Sensores LM 35 inalámbricos, ubicados en los Sectores descriptos sobre la Fachada Experimental, Figura 4.

Cabe mencionar que en este trabajo, el indicador experimental de las mejoras producidas en la fachada, está representado por las diferencias de temperaturas del revoque externo con módulo respecto del revoque externo expuesto.

A las $12 \mathrm{~h}$, con una Radiación Solar de $830 \mathrm{~W} / \mathrm{m}^{2}$ y $47^{\circ} \mathrm{C}$ de temperatura de bulbo seco, con el SPETS ventilado, mientras se producían diferencias de hasta $15,5^{\circ} \mathrm{C}$, considerando 


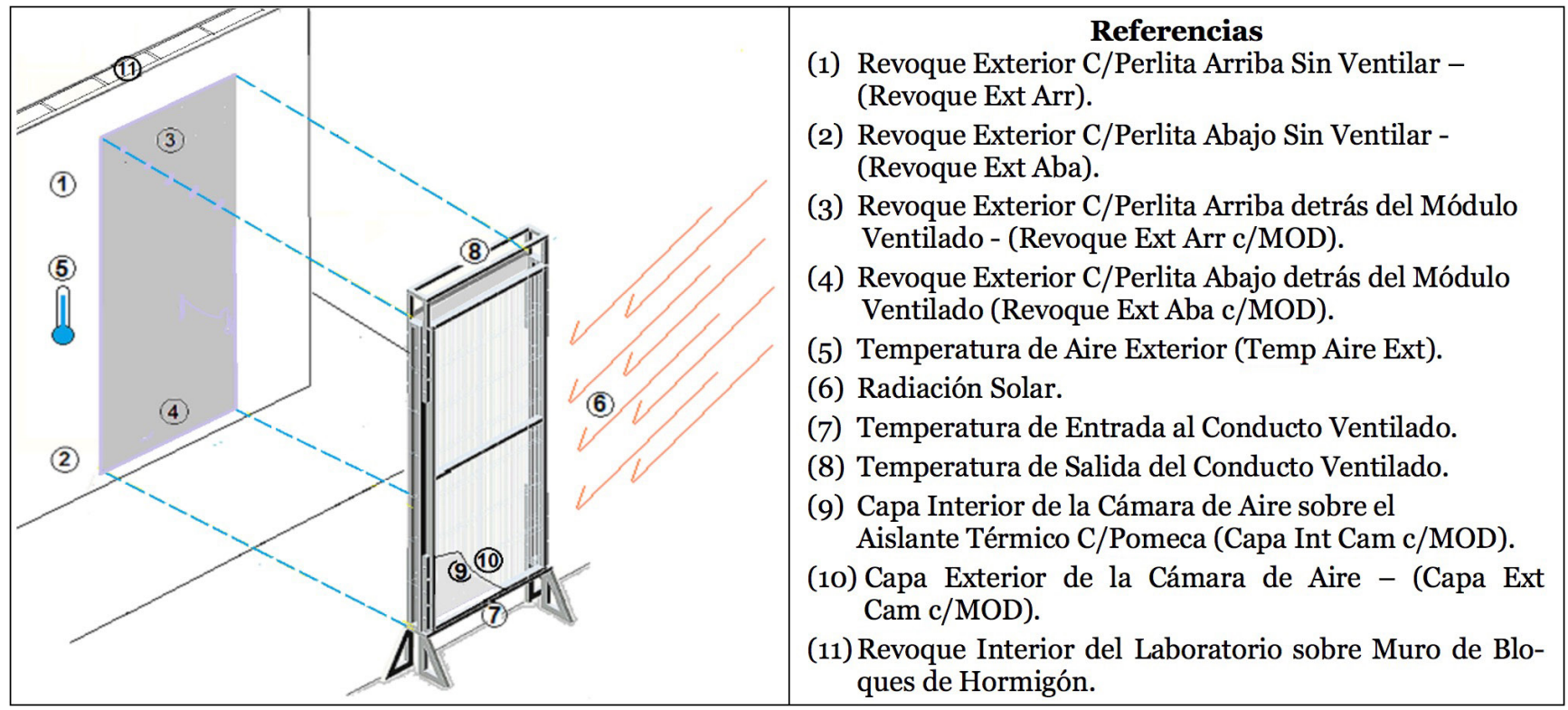

Figura 4: Esquema de distribución de sensores y referencias de los parámetros térmicos sensados.
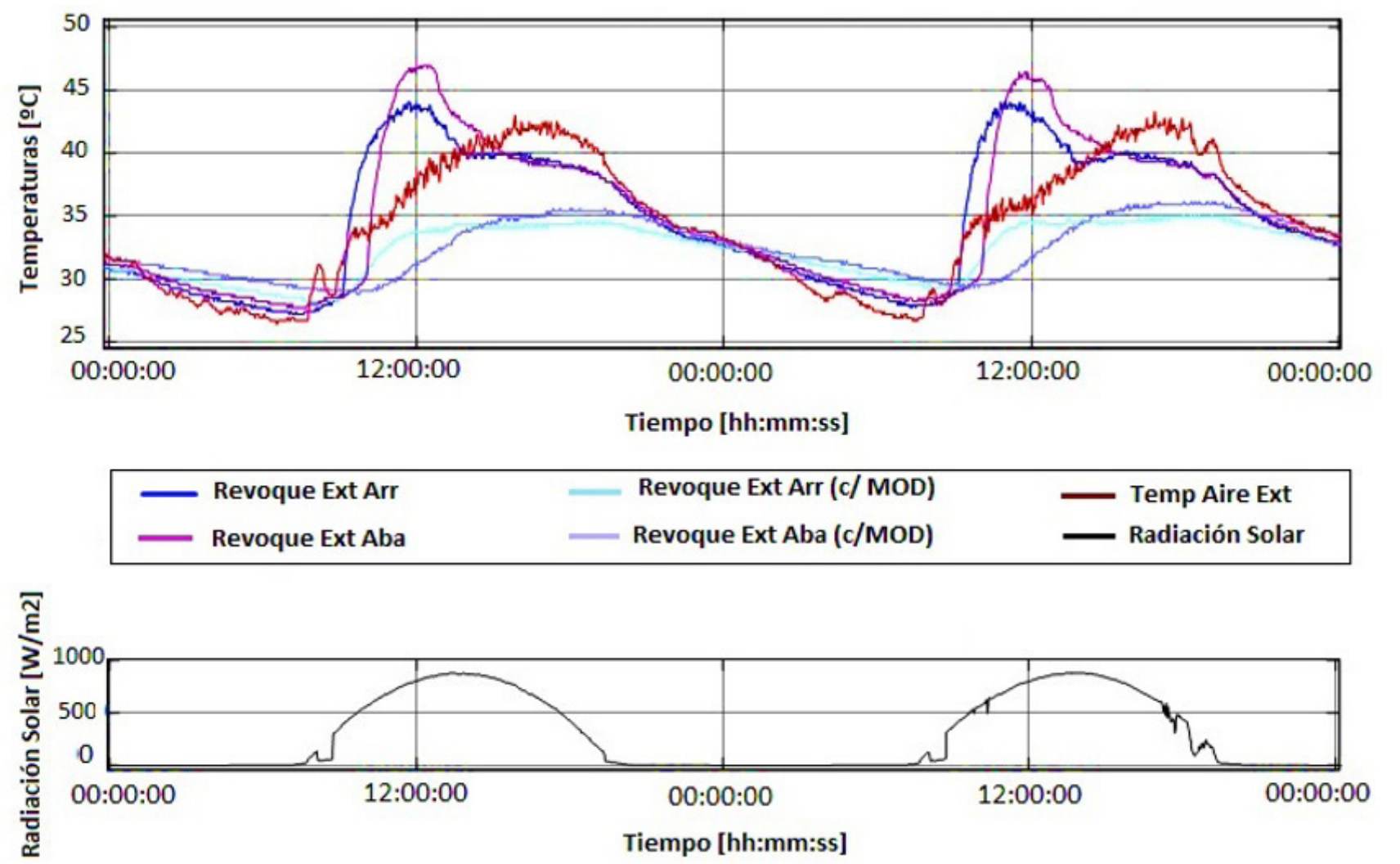

Figura 5: Comportamiento en Verano de la Fachada Ventilada (c/MOD) y sin Ventilar. Fuente: Elaboración del Autor.

la Temperaturas de Entrada y la de Salida del conducto de aire. Se obtuvieron diferencias de $16^{\circ} \mathrm{C}$ entre ambos sectores de la fachada, debido a que en el Revoque Exterior C/ Perlita Abajo detrás del Módulo ventilado se alcanzó $31,5^{\circ} \mathrm{C}$. Y en el Revoque Exterior C/ Perlita Abajo de la fachada sin ventilar, que solo presenta el revoque expuesto al ambiente exterior sin cámara se llegó a obtener $46,5{ }^{\circ} \mathrm{C}$, Figura 5 .

Y en la campaña de mediciones representativa de la época invernal de la ciudad de San Juan, realizada entre el 17 y el
20/06/2016 inclusive, se verificó en el área cubierta por el Módulo aislado y con Cámara de Aire Sin Ventilar una variación o amplitud térmica de casi $18^{\circ} \mathrm{C}$, entre el exterior y el interior.

Esto puede observarse en la Figura 6 durante el segundo día de medición, a las 07:51h, cuando para una Temperatura Mí$\operatorname{nima}\left({ }_{\mathrm{M}}\right)$ de Aire Exterior de $4.75^{\circ} \mathrm{C}$, Humedad Relativa del $88 \%$ y viento en calma se registraban a Nivel Inferior, en la Capa Externa de la Cámara de Aire $4.12^{\circ} \mathrm{C}$ y en la Tempera- 
tura del Revoque Interior del Laboratorio dispuesto sobre el muro de bloques de hormigón $22.15^{\circ} \mathrm{C}$, Figura $2 \mathrm{~b}$. A la vez en la Capa Interior de la Cámara de Aire sobre el Aislante Térmico (Pomeca) se llegaba a los $7.18^{\circ} \mathrm{C}$ y en el Revoque Exterior con Perlita detrás del Módulo Sin Ventilar, a los $16.62^{\circ} \mathrm{C}$.

Mientras que en el sector del muro sin el Módulo aislante se obtuvo una variación térmica menor a $9^{\circ} \mathrm{C}$, como diferencia entre los $7.12^{\circ} \mathrm{C}$ del Revoque Exterior sin módulo y los $16.09^{\circ} \mathrm{C}$ del Revoque Interior del Laboratorio, Figura 2b. Por otro lado de acuerdo a las experiencias de invierno, se obtuvieron a Nivel Inferior $9,5^{\circ} \mathrm{C}$ de diferencia, entre el Revoque Exterior detrás del Módulo Sin Ventilar y el Revoque Exterior con Perlita, Figura 4.

Cabe señalar que la cámara de aire fue incorporada para ventilar la fachada en verano y así facilitar las pérdidas de calor por convección, radiación y conducción limitando su transferencia hacia el interior.

Por otro lado se ensayaron las características ignífugas modulares del SPETS, utilizando fijaciones estructurales, aplicadas por el exterior del laboratorio IMA-AERA-FI-UNSJ que presenta un entorno abierto $1500 \mathrm{~m}^{2}$, en la que se ha planificado la reducción al mínimo de los efectos de vientos de moderados a catastróficos.

En esta interface el sistema constructivo del SPETS que fuera fijado a los muros del Laboratorio a través de su capa de nivelación termoaislante soporto la incidencia y exposición directa a severos de vientos Zondas y Sur, tal como el del 21 de julio de 2019, detallado en el apartado 2.1.1.

Este viento Zonda presentó numerosas novedades, que incluyo 55 incendios producidos a nivel de la interface mediata $\mathrm{e}$ inmediata, forestal, con alto impacto en viviendas, y personas en la región árida de Mendoza, Tabla 1. Y que también contara con similares novedades en San Juan.

Durante el periodo de más de 4 años de ensayos, el SPETS fue expuesto a la acción directa al Clima Árido en forma permanente, y ha soportado un gran número de Vientos Zonda y Sur que se han caracterizado principalmente por la variabilidad de sus ráfagas.

Esta ráfagas oscilaron entre 60 y $120 \mathrm{~km} / \mathrm{h}$, con cambios abruptos en la temperatura de bulbo seco y en la temperatura de rocío, con aumentos del orden de hasta $40^{\circ} \mathrm{C}$, con $3-5^{\circ} \mathrm{C} /$ hora y disminuciones del orden de $10-20^{\circ} \mathrm{C} /$ hora respectivamente con la llegada de vientos frescos provenientes del cuadrante sur-sureste.

Cabe destacar en relación a su resistencia al calor al fuego, además de la alta presión y succión producidas, que las estrategias de seguridad adoptadas, han preservado al SPETS y al Laboratorio durante la incidencia de la rafagosidad descripta, del desarrollo de chispas y fuego, debido a los materiales y sistema constructivo ignífugos que incluye las fijaciones em-
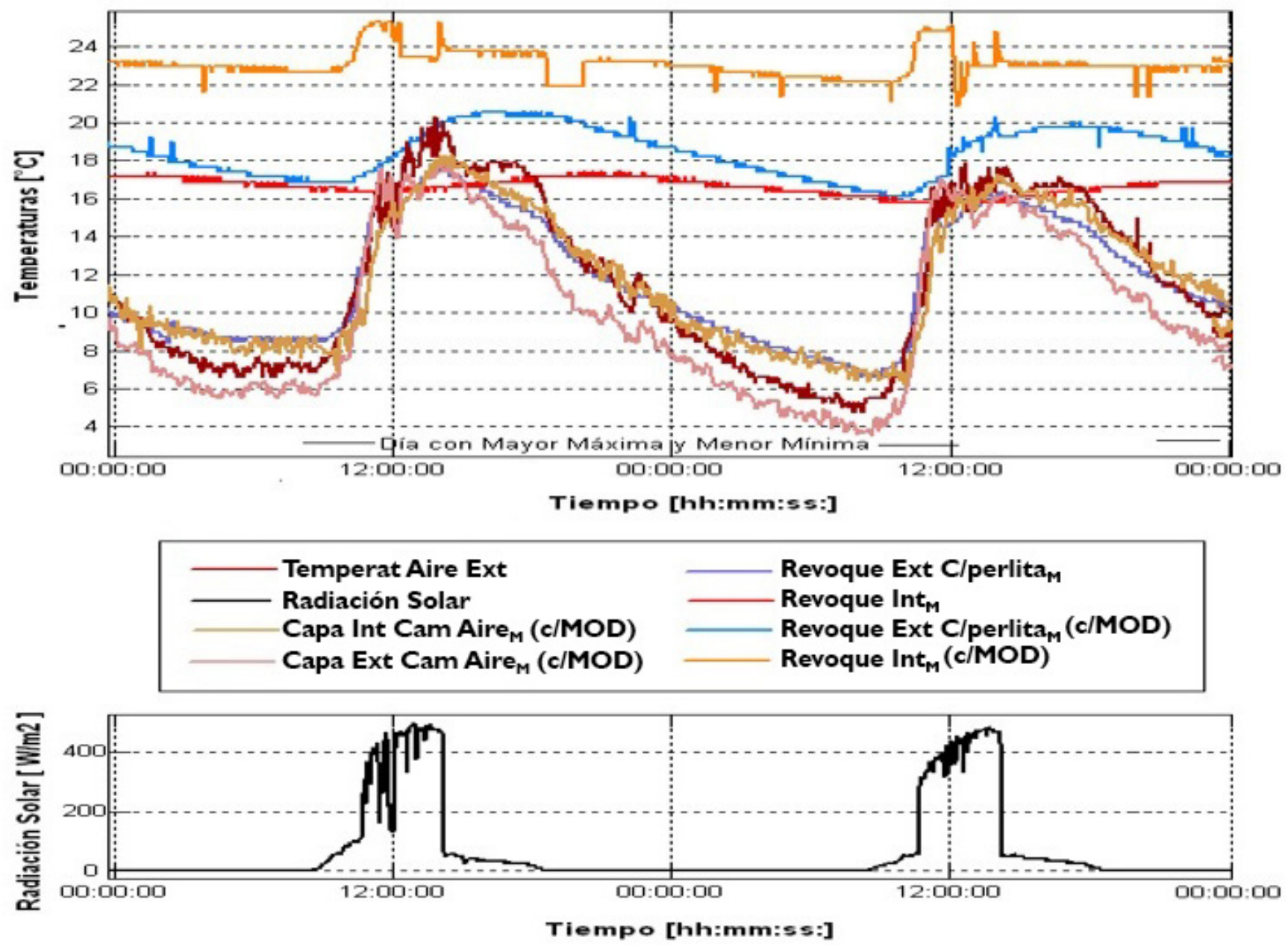

Figura 6: Comportamiento térmico en Invierno de la Fachada con Módulo sin Ventilar y sin Módulo. Fuente: Elaboración del Autor 
pleadas, así como también la planificación de la hermeticidad del conjunto.

\section{CONCLUSIONES}

El diseño abierto e integral del soporte estructural del SPETS, junto a las alternativas estudiadas incluyendo PPZ, contribuyen a mejorar el confort higrotérmico interior de Barrios Construidos, con cumplimiento de las Normas Argentinas. Demuestran brindar adecuadas respuestas a requerimientos de aislación de muros con elevada carga térmica por orientaciones desfavorables, se adapta a cambios o crecimientos futuros, a su incorporación de acuerdo a la disponibilidad de espacios en planta y a diferentes tipos de aislantes reciclables naturales de origen regional.

Con la implementación experimental del módulo se logró disminuir los gradientes de temperaturas entre el revoque exterior, respecto a los gradientes ocurridos en el muro directamente expuesto al ambiente exterior. Dichas mejoras en los gradientes reducen la transferencia de calor hacia el interior del Box Experimental del IMA.

Las reducciones en la transferencia de calor alcanzadas con la utilización con el SPETS durante el periodo estival, harán posible climatizar los interiores con menores consumos de energía. Por otro lado, al acumularse menores cantidades de energía durante el día, tanto en las envolventes como en el aire interior, se disminuirá el consumo energético necesario para extraer dicho calor acumulado en las noches, mediante el aprovechamiento del aire ambiente exterior a temperaturas de confort, utilizando sistemas de refrescamientos naturales pasivos e híbridos.

Por ello en un futuro trabajo y con el objetivo de poder evaluar la eficacia energética anual del sistema en forma integral, se desarrollará el estudio correspondiente a la implementación conjunta del SPETS junto a estrategias de climatización natural.
La prefabricación del SPETS responde a un diseño modular en el que se han considerado el menor impacto ambiental de sus partes y componentes. Este menor impacto incluye la planificación ignífuga del conjunto expuesto a los efectos de vientos secos de gran magnitud con riesgo de incendio.

El armado en la industria evita retrasos de mano de obra por mal tiempo, responde a una estandarización de procesos que incluye la construcción de multicapas termoaislantes aplicadas "en seco" con la utilización de componentes livianos, móviles y desarmables compatibles con la incorporación a granel de un material regional aislante e ignifugo de origen volcánico (PPZ).

La transportabililidad del SPETS desde la metalúrgica, y su instalación sencilla contribuye a la reducción de costos de mano de obra, como también facilita tanto la inserción como el desarme y la reutilización, según las necesidades en distintos contextos sociales urbanos.

En este sentido la aplicación del SPETS resulta útil para paliar la crítica situación actual energética y económica, de la Zona Árida de Cuyo, que se encuentra afectada por la necesidad de ahorro energético en más de 400.00o VDSC.

\section{AGRADECIMIENTOS}

Se agradece al Ing. Leonardo A. Pogrebinsky integrante del Área de Energías Renovables y Ambiente (AERA) Instituto de Mecánica Aplicada (IMA) - Facultad de Ingeniería (FI) - Universidad Nacional de San Juan (UNSJ), por su contribución en la obtención de sensados de comportamiento térmico del SPETS.

También se agradece al personal no docente del IMA-FI por su colaboración en trabajos metalúrgicos y de campo. Además se agradece a la Secretaría de Ciencia y Técnica - CICITCA - Rectorado-UNSJ, por la Aprobación y Financiación de este proyecto.

\section{REFERENCIAS}

(1) PNUD (2016). Agenda 2030 y los Objetivos de Desarrollo Sostenible. Una oportunidad para América Latina y el Caribe. Publicación de las Naciones Unidas. Santiago, Chile.

(2) PLAN NACIONAL DE VIVIENDA SOCIAL (2017). Línea de Acción 1 - Promoción de la Vivienda Social. Manual de Ejecución. Y Línea de Acción 2 y 3 - Estándares Mínimos de Calidad para Viviendas de Interés Social - Revisión 2017. Dirección de Tecnología y Producción Dirección Nacional de Desarrollo Urbano y Vivienda. Subsecretaría de Desarrollo Urbano y Vivienda. Secretaría de Vivienda y Hábitat. Ministerio del Interior, Buenos Aires, Argentina.

(3) Mercado M. V., Esteves A., Filippín C. (2010). Comportamiento térmico-energético de una vivienda social de la ciudad de Mendoza, Argentina. Laboratorio de Ambiente Humano y Vivienda. Centro de Ciencia y Técnica Consejo Nacional de Investigaciones Científicas y Técnicas. Ambiente Construído. On-line version ISSN 1678-8621. vol.10 no.2 Porto Alegre Apr./June 2010. https://doi.org/10.1590/S1678-86212010000200006 Mendoza, Argentina.

(4) Viegas G. M., Walsh C., Barros M. V. (2016). Evaluación cuali-cuantitativa de aislaciones térmicas alternativas para viviendas. El caso de la agricultura familiar. Revista INVI vol.31 no.86. http://dx.doi.org/10.4067/So71883582016000100004. Santiago, Chile.

(5) Sans J. (2019). Estudio sobre la Problemática Generada por la Propagación de Incendios en Fachadas de Edificios. Collegi/Associació d'Enginyers Industrials de Catalunya. Collegi Oficial d'Enginyers Industrials de Catalunya (COEIC). Documento - Enero 2019, pp9-10. Barcelona, España.

(6) Torrijos P. (2017). ¿Porque ardió Grenfell Tower?. Diario el Economista. Editorial Ecoprensa. España.

(7) Buigues Nollens A. (2013). Aprovechamiento de la Pomeca Puzolánica en Envolventes. : Desarrollo Experimental de un Panel Termoaislante para Viviendas en Zonas Áridas en Argentina. Revista Hábitat Sustentable, Vol. 3 N ${ }^{\circ} 1$, pp.6271. ISSN: 0719 - 0700. Universidad del Bio Bio, Chile.

(8) García -Figueruelo B., Valcuende M., Bonilla M., Borrachero V., Payá J., Buigues-Nollens A., Monzó J. (2017). ¿Puede la Pomeca Argentina Contribuir a la Mejora de la Calidad de la Vivienda de la Población de Menores Recursos? Libro de Actas del III Congreso Internacional de Estudios del Desarrollo, línea temática 6: Desarrollo sostenible. Tecnologías para un Desarrollo Humano, Zaragoza España, pp.457-467. ISBN: 978-84-16723-36-2. Instituto de Ciencia y Tecnolo- 
gía del Hormigón, (Icitech-Upv); Depto. de Construcciones Arquitectónicas. Universitat Politècnica de València España e Instituto de Mecánica Aplicada. Facultad de Ingeniería. Universidad Nacional de San Juan. Argentina.

(9) Azqueta P. (2017). Manual Práctico del Aislamiento Térmico. AAPE Asociación Argentina del Poliestireno Expandido para una Construcción Sustentable. Cuarta Parte 04, Soluciones constructivas para paredes y techos. Segunda Edición. Argentina.

(10) IRAM 11603 (1996). Acondicionamiento Térmico de Edificios. Clasificación bioambiental de la Argentina, p.1- 47. , Argentina.

(11) Otero F., Norte F. A. (2015). Métodos de Clasificación y Climatología del Viento Zonda en San Juan. Asociación Argentina de Geofísicos y Geodestas. Instituto Argentino de Nivología, Glaciología y Ciencias Ambientales, Centro Regional de Investigaciones Científicas y Técnicas/CONICET. GEOACTA 4O(1): pp.45-53. ISSN 1852-7744. Mendoza, Argentina.

(12) Norte, Federico Augusto 1988. Características del viento zonda en la región de Cuyo Tesis presentada para obtener el grado de Doctor en Ciencias Meteorológicas de la Universidad de Buenos Aires da en la región de Cuyo. Facultad de Ciencias Exactas y Naturales. Universidad de Buenos Aires. http://digital.bl.fcen.uba.ar/Download/Tesis/Tesis_2131_ Norte.pdf. Argentina.

(13) Valle R. (2019). El Zonda desnudo la fragilidad del arbolado público de la provincia. Diario de Mendoza On line. 24 de julio de 2019. http://www.mdzol.com. Mendoza, Argentina.

(14) Pau Costa Foundation, (2014). Los incendios forestales en Interfaz urbana: Hacia la integración del riesgo en la planificación del territorio. Conclusiones de las Jornadas Euromediterránea en Incendios Forestales. Barcelona, España.

(15) Fiscarelli D. M., Cortina, K. A. (2013). La adaptabilidad como variable de la calidad de la vivienda. Vivienda, adaptabilidad y criterios proyectuales. $1^{\circ}$ Congreso Patología, control de calidad y rehabilitación en la construcción, p.12-17. Argentina.

(16) Gelabert Abreu D. y González Couret D. (2013). Vivienda progresiva y flexible. Aprendiendo del repertorio. Arquitectura y Urbanismo, Vol.34, $\mathrm{N}^{0} 2$. p. 48-63, ISSN 1815-589. La Habana, Cuba.

(17) Salas, G. (2011). IRAM K. Software de Diseño Higrotérmico basado en Normas IRAM 11601, 11605,11625 y 11630. Revisado en la Universidad Nacional de Mar del Plata, Buenos Aires, Argentina.

(18) Cremaschi G., Marsili L., Saenz A. (2013). Acondicionamiento Higrotérmico de Edificios. Taller Vertical de Procesos Constructivos. PC 1 Ficha $\mathrm{N}^{\circ}$ 03. Manual de Aplicación Ley 1359. pp. 13 a 36. Argentina.

(19) IVE (2011). Productos y Materiales. Propiedades de Aislantes Térmicos para Rehabilitación Energética. Cuadernos de Rehabilitación. Instituto Valenciano de la Edificación. España, pp.3.

(20) Olalla B. M. (2014). Fachadas Sostenibles. Proyecto de graduación presentado para la carrera de Ingeniería Civil de la Escuela Politécnica, como parte de los requisitos necesarios para la obtención del título de Ingeniería Civil., Universidad Federal de Río de Janeiro, Brasil, 2014.

(21) Maroto P. (2014). Mejora energética de la envolvente. Fachada ventilada o sin ventilar. Comunicación presentada al II Congreso Edificios Energía Casi Nula. Knauf GmbH. España.

(22) IRAM 11605 (2002). Acondicionamiento térmico de edificios. Condiciones de habitabilidad en edificios. Valores máximos de transmitancia térmica en cerramientos opacos. Puentes térmicos. Revisión de la norma IRAM 11605:1996, p.8-20, Argentina.

(23) Pascual Román N. (2014). La Eficiencia Energética en el Uso de la Vivienda. Factores Incidentes. Trabajo de Fin de Master Universidad Politécnica de Valencia, p.40-62. España.

(24) Berardi R. (2014). Reciclado Energético de la Envolvente de Vivienda Unifamiliar en La Plata, Argentina. Revista Avances en Energías Renovables y Medio Ambiente AVERMA. Vol. 18, pp.05.29-05.38, 2014. ISBN 978-987-29873-0-5. Argentina.

(25) Buigues Nollens A. y Pogrebinsky Mazarico L. (2014). Paneles Termoaislantes con Ecodiseño para Viviendas Sociales de Zonas Áridas. $1^{\circ}$ Congreso Latinoamericano de Ecodiseño - Ecodal 2014. Vol.1, No 1. ISSN o719-577X. Santiago, Chile.

(26) INPRES (2013). Zonificación Sísmica, Nuevo Reglamento INPRES CIRSOC 2013. Reglamento Argentino para Construcciones Sismorresistentes. Instituto Nacional de Prevención Sísmica Secretaría de Obras Públicas. Ministerio de Planificación Federal, Inversión Pública y Servicios. Parte 1, p.13-45. Argentina. 